\title{
Anabolic Activity of a Soy Extract and Three Major Isoflavones in C2C12 Myotubes
}

Authors

Wenya Zheng ${ }^{1}$, Marie L. Hemker ${ }^{1}$, Mingyong $\mathrm{Xie}^{2}$, Sebastian T. Soukup ${ }^{3}$, Patrick Diel ${ }^{1}$

Affiliations

1 Institute of Cardiovascular Research and Sports Medicine, Department of Molecular and Cellular Sports Medicine, German Sport University Cologne, Germany

2 State Key Laboratory of Food Science and Technology, Nanchang University, Nanchang, China

3 Department of Safety and Quality of Fruit and Vegetables, Max Rubner-Institut, Karlsruhe, Germany

Key words

isoflavones, Glycine max, Leguminosae, C2C12 myotube, IGF-1, MHC

\author{
received October 18, 2017 \\ revised March 6, 2018 \\ accepted March 26, 2018
}

Bibliography

DOI https://doi.org/10.1055/a-0598-4812

Published online April 12, 2018 | Planta Med 2018; 84: 10221029 @ Georg Thieme Verlag KG Stuttgart · New York | ISSN 0032-0943

Correspondence

Prof. Patrick Diel

Institute of Cardiovascular Research and Sports Medicine, Department of Molecular and Cellular Sports Medicine, German Sport University Cologne

Am Sportpark Muengersdorf 6, 50933 Cologne, Germany Phone: +49(0)22149825860, Fax: +49(0)22149828370 diel@dshs-koeln.de

\section{ABSTRACT}

Isoflavones have been reported to stimulate muscle growth. The aim of this in vitro study was to examine anabolic activity and associated molecular mechanisms of a soy extract (SoyEx), isoflavone aglycones, and a mixture simulating the composition of SoyEx in C2C12 myotubes. C2C12 cells were differentiated into myotubes. The effects of SoyEx, genistein, daidzein, glycitein, and the mixture of genistein-daidzein-glycitein (Mix) on myotube diameter and number were determined. In addition, the expression of genes and proteins associated with anabolic activity was analyzed. Treatment with SoyEx, genistein, and Mix led to a significant increase of myotube diameter and an increase of the number of myotubes per area compared to the control cell. The increase of diameter by SoyEx was antagonized by an antiestrogen, not by an antiandrogen. Furthermore, gene expressions of insulin growth factor (IGF)-1 and its receptor (IGF-1R), as well as protein expression of myosin heavy chain (MHC), were significantly increased by SoyEx, genistein, and Mix. The effects induced by genistein and Mix were comparable to SoyEx. In conclusion, SoyEx displays an anabolic activity in C2C12 myotubes by binding to ER and modulating IGF-1 and MHC expression. Our studies with isoflavone aglycones and Mix indicate that the isoflavone aglycone with the highest anabolic bioactivity in SoyEx is genistein.

\section{Introduction}

Skeletal muscle accounts for the most mass of an individual and daily energy consumption. It is able to respond and adapt to changing environmental stimuli. Endocrine factors influence muscle growth and development throughout life. Increasing evidence shows that menopause is associated with a decline in muscle mass and muscle strength [1], indicating a correlation between muscle mass and estrogen status in females [2]. For these reasons, HRT is regarded as a therapeutic strategy for women who are suffering the loss of muscle mass and muscle function. However, HRT has a complex pattern of risks and benefits $[3,4]$. Isoflavones are naturally occurring diphenolic compounds ( $\bullet$ Fig. 1 A) and are discussed as an alternative for HRT because of their similar chemical structure to E2. Soy (Glycine max [L.] Merr., Leguminosae) is the main source for isoflavones [5]. Aubertin-Leheudre et al. [6] showed that lean body mass and muscle mass of obese-sarcopenic postmenopausal women were significantly increased after supplementation of $70 \mathrm{mg}$ isoflavones per day for $24 \mathrm{wk}$. Besides genistein, daidzein, and glycitein, which are the principal isoflavones found in soy foods, there are also other chemicals identified in soy products, such as genistin, daidzin, glycitin. However, genistein has been shown to exhibit greater biological activity than its beta-glucoside conjugate genistin $[7,8]$.

Skeletal muscle is composed of multinucleated fibers that are formed after myoblast differentiation and fusion together. The fi- 


\begin{tabular}{ll} 
ABBREVIATIONS \\
AR & androgen receptor \\
DHT & dihydrotestosterone \\
E2 & 17ß-estradiol \\
ER & estrogen receptor \\
HRT & hormone replacement therapy \\
IGF & insulin-like growth factor \\
MHC & myosin heavy chain \\
Mix & a mixture of genistein-daidzein-glycitein \\
mTOR & mammalian target of rapamycin \\
MuRF1 & muscle RING finger-containing protein 1 \\
PKB & protein kinase B \\
PI3K & phosphatidylinositol 3-kinase \\
SoyEx & a soy extract \\
& \\
\hline
\end{tabular}

bers are analogous to myotubes in vitro. C2C12 cells, a myoblast cell line derived from murine satellite cells, have been used extensively as an in vitro model to study both muscle differentiation and hypertrophy [9]. Differentiated myotubes of C2C12 cells exhibit a hypertrophic response to growth factors like IGF-1 and E2, characterized by changes of diameters in myotubes and an activation of protein synthesis [9]. Previously, our group demonstrated that $E R \beta$ is involved in the hypertrophy of $\mathrm{C} 2 \mathrm{C} 12$ myotubes induced by the phytoecdysteroid ecdysterone [10]. Another in vivo study in our group showed that genistein is able to affect muscle growth through ER $\beta$ signaling pathway, showing an increased expression of MHC [11]. MHC determines muscle fiber type and contractile speed based on ATPase properties in skeletal muscle. In mammals, several isoforms of MHC proteins are expressed in the sarcomere, displaying distinct contractile properties depending on the composition of muscle fibers. An in vivo study showed that $\mathrm{MHC}$ composition was shifted from fast to slow MHC isoform in soleus and extensor digitorum longus by ovariectomy in rats [12]. In C2C12 neo-formed myotubes, MHC is a major structural protein and acts as a differentiation marker [13]. Additionally, MyoD, which belongs to a family of proteins known as myogenic regulatory factors, is a protein that plays a major role in regulating muscle differentiation. Moreover, IGF-1 is among the best-characterized factors involved in muscle hypertrophy. IGF-1 directly interacts with its own receptor, IGF-1R, followed by activating the PI3K/Akt pathway [14].

In this study, our major aim was to analyze the effect of SoyEx on hypertrophy of C2C12 myotubes. We compared the biological activity of SoyEx to the activities of its three major isoflavone compositions (genistein, daidzein, glycitein) and Mix simulating the composition in SoyEx. Moreover, the effects of SoyEx and isoflavones on molecular mechanisms related to the hypertrophy of C2C12 myotubes were investigated.

\section{Results}

SoyEx contained about $5.3 \mathrm{mg} / \mathrm{g}$ genistein, $10.4 \mathrm{mg} / \mathrm{g}$ daidzein, and $3.2 \mathrm{mg} / \mathrm{g}$ glycitein (data were shown in our previous paper) [15]. Other isoflavone derivatives (i.e., genistin, 6"'-O-acetyl-gen- istin, daidzin, 6"-O-acetyl-daidzin, glycitin, 6"'-O-acetyl-glycitin) were also detected (data were shown in our previous paper) [15]. A representative HPLC-UV chromatogram of SoyEx showing the identified isoflavone peaks is illustrated in $>$ Fig. $\mathbf{1 B}$.

The effect of $48 \mathrm{~h}$ treatment of SoyEx on the diameter of myotubes is shown in $>$ Fig. 2. As shown in > Fig. 2 A, different dilutions of SoyEx $(0.0005-50 \mu \mathrm{g} / \mathrm{mL})$ resulted in a bell-shaped doseresponse curve, inducing a significant increase in myotube diameter at a concentration of $0.05 \mu \mathrm{g} / \mathrm{mL}$. The increase was similar to that induced by $\mathrm{E} 2$ at a concentration of $10 \mathrm{nM}(\triangleright$ Fig. $2 \mathrm{~A}, \mathrm{C}$ ). Further, the diameters of $\mathrm{C} 2 \mathrm{C} 12$ myotubes treated with genistein $(0.001 \mu \mathrm{M})$, daidzein $(0.0021 \mu \mathrm{M})$, and glycitein $(0.0006 \mu \mathrm{M})$, which were at the same concentrations as determined in $0.05 \mu \mathrm{g} / \mathrm{mL}$ SoyEx, were measured ( $\triangleright$ Fig. 2 B). The Mix was also composed of genistein, daidzein, and glycitein, which have exactly the same concentrations as in $0.05 \mu \mathrm{g} / \mathrm{mL}$ SoyEx. Treatment of genistein and Mix led to significant increases of the diameters, which was comparable to the effect induced by SoyEx.

The effect of the respective substances on the number of myotubes in a defined area is shown in $>$ Fig. 3, - Fig. $\mathbf{3}$ A and $\mathbf{B}$ show the same area of a six-well plate before and after $48 \mathrm{~h}$ treatment with $0.05 \mu \mathrm{g} / \mathrm{mL}$ of SoyEx. It is clearly visible that the treatment with SoyEx resulted in an increase of the myotube diameter; however, the number of myotubes in this area did not change after $48 \mathrm{~h}$ treatment. Comparing the number of myotubes per area among the treatment groups ( $\bullet$ Fig. $3 \mathbf{C}$ ) revealed treatment with all substances resulted in a significantly higher number of myotubes in the treated groups compared to the control group.

The relevance of ER and AR in the anabolic effect of SoyEx is shown in $>$ Fig. 4. As shown in $>$ Fig. $4 \mathbf{A}$, the increased diameter induced by the treatment of either E2 or SoyEx for $48 \mathrm{~h}$ was antagonized by an antiestrogen ZK191703. In contrast, a selective antagonist of AR flutamide significantly antagonized the myotube hypertrophy induced by DHT, whereas it was not able to decrease the diameter when cotreated with SoyEx ( $\vee$ Fig. 4B).

The mRNA expressions of IGF-1, IGF-1R, and MuRF1 in C2C12 myotubes after $48 \mathrm{~h}$ treatment are shown in > Fig. 5. IGF-1 expression ( $\bullet$ Fig. 5A) was significantly increased by E2, SoyEx, genistein, or Mix. There were no significant differences found between either E2 and SoyEx, between SoyEx and genistein, or between genistein and Mix. Also, IGF-1R expression ( $\bullet$ Fig. 5B) was increased by E2, SoyEx, genistein, or Mix in C2C12 myotubes, which was similar as regulated with IGF-1. However, the expression of MuRF1 ( $\vee$ Fig. $\mathbf{5 C}$ ) was not affected by any of the treatments.

The protein expressions of $\mathrm{MHC}$ and MyoD in C2C12 myotubes after $48 \mathrm{~h}$ treatment are shown in $\triangleright$ Fig. 6. As shown in $\triangleright$ Fig. 6A, E2, SoyEx, genistein, daidzein, and Mix significantly increased $\mathrm{MHC}$ protein expression and the highest expression was detected in the E2 group. The effect of genistein was similar to SoyEx. Moreover, MyoD protein expression ( $\triangleright$ Fig. 6B) was not affected by any of the treatments. 
A
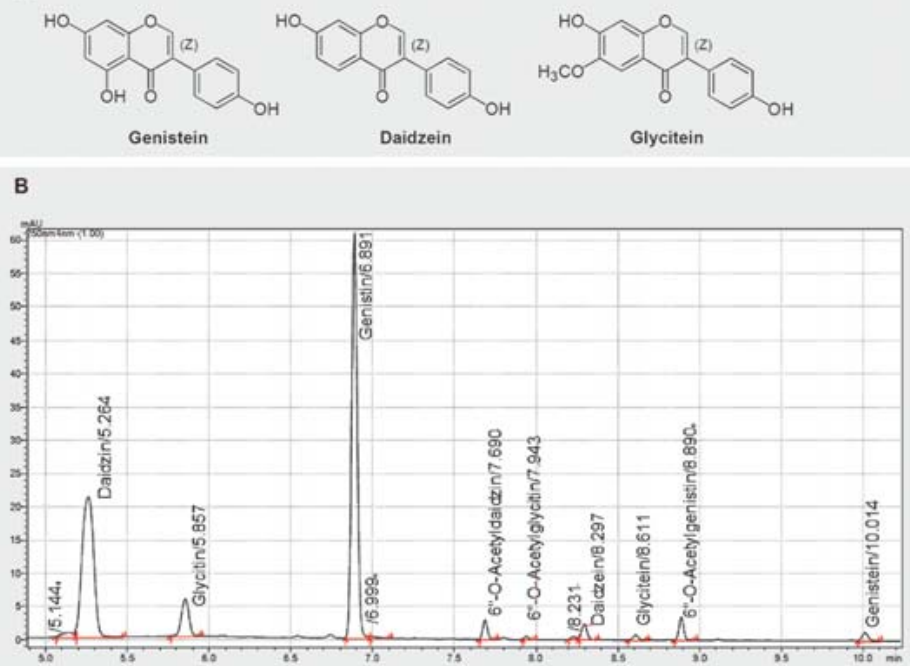

- Fig. 1 Chemical structure and a detailed chemical characterization SoyEx. A The chemical structure of genistein, daidzein, and glycitein. B A representative HPLC-UV chromatogram $(250 \mathrm{~nm})$ of SoyEx showing the identified isoflavone peaks.

A C2C12 cells treated with Soy extract

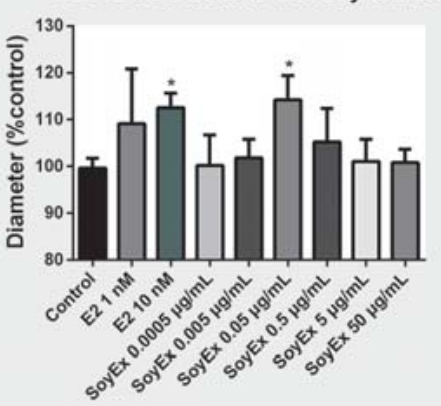

B

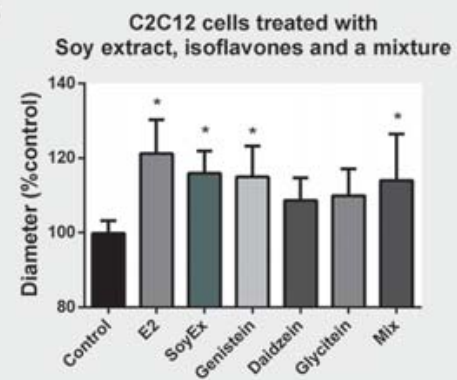

- Fig. 2 Diameter measurement of C2C12 myotubes treated with increasing doses of SoyEx (A) and genistein $(0.001 \mu \mathrm{M})$, daidzein $(0.0021 \mu \mathrm{M})$, and glycitein $(0.0006 \mu \mathrm{M})$ at concentrations the same as determined in $0.05 \mu \mathrm{g} / \mathrm{mL}$ SoyEx, as well as Mix $(0.001 \mu \mathrm{M}, 0.0021 \mu \mathrm{M}, 0.0006 \mu \mathrm{M})(\mathrm{B})$. C Differences of the diameters in control, E2 $(10 \mathrm{nM})$, and SoyEx $(0.05 \mu \mathrm{g} / \mathrm{mL})$ groups. The diameter of control group was set as $1 .{ }^{*} \mathrm{p} \leq 0.05 \mathrm{vs}$. control. 
A

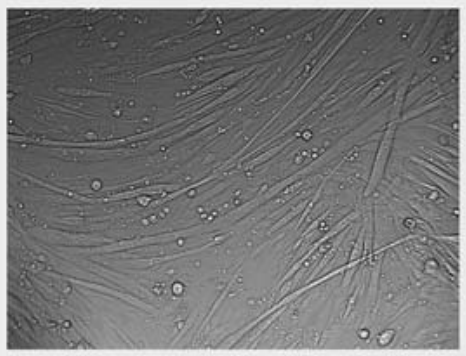

B

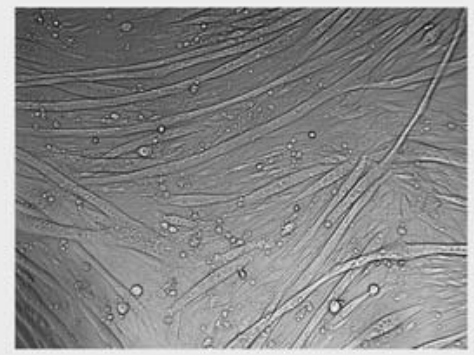

C2C12 cells treated with

C

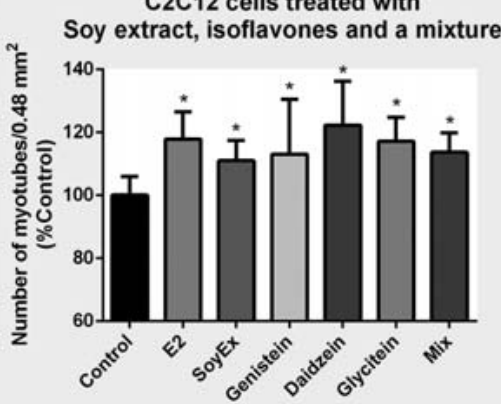

Fig. 3 Measurement of C2C12 myotube number in specific areas. A, B The same area on a 6-well plate before and $48 \mathrm{~h}$ after the treatment with SoyEx $(0.05 \mu \mathrm{g} / \mathrm{mL})$. C The number of myotubes in a $0.48 \mathrm{~mm}^{2}$ area after $48 \mathrm{~h}$ incubation with the respective substances. $\mathrm{E} 2=10 \mathrm{nM}$, SoyEx $=$ $0.05 \mu \mathrm{g} / \mathrm{mL}$, genistein $=0.001 \mu \mathrm{M}$, daidzein $=0.0021 \mu \mathrm{M}$, glycitein $=0.0006 \mu \mathrm{M}, \mathrm{Mix}=(0.001 \mu \mathrm{M}, 0.0021 \mu \mathrm{M}, 0.0006 \mu \mathrm{M})$. The number of control group was set as $1 .{ }^{*} \mathrm{p} \leq 0.05$ vs. control.

A

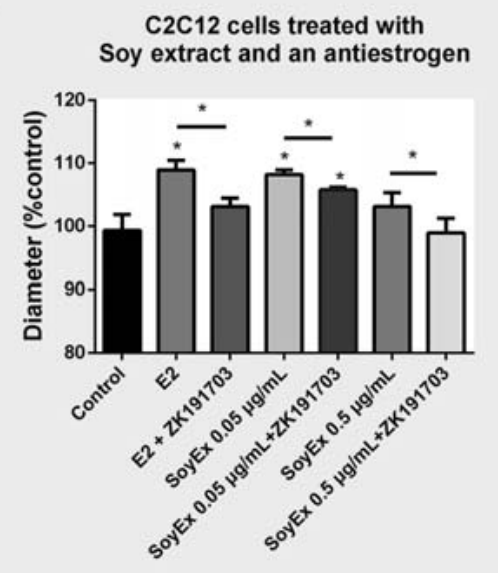

B

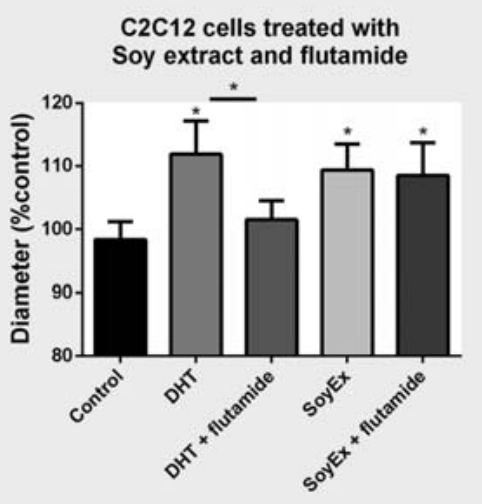

- Fig. 4 Investigation of the role of AR (A) and ER (B) in affecting the increases of diameters of C2C12 myotubes that treated with SoyEx. $\mathrm{E} 2=10 \mathrm{nM}$, SoyEx $=0.05 \mu \mathrm{g} / \mathrm{mL}, \mathrm{ZK} 191703=1 \mu \mathrm{M}$, flutamide $=1 \mu \mathrm{M} .{ }^{*} \mathrm{p} \leq 0.05 \mathrm{vs}$. control.

\section{Discussion}

Skeletal muscle growth, also known as hypertrophy, is characterized as an increase in muscle mass and myofiber size, which can be induced by anabolic hormonal substances (i.e., DHT and E2) [10]. C2C12 myoblastoma cells differentiated into myotubes is an accepted cell culture model to study anabolic activity [9]. Previously, studies of our group using a model of C2C12 myotubes demonstrated that ecdysterone affects muscle growth via ER-mediated mechanisms [10]. In this study, we could demonstrate a dose-dependent anabolic activity of SoyEx on C2C12 myotubes, which is quantitatively comparable to the effects observed for E2 at $10 \mathrm{nM}$ ( $\vee$ Fig. 2A). Information about anabolic effects of SoyEx on skeletal muscle cells is limited. In rat L6 skeletal muscle cells, genistein promoted cells proliferation at doses $\leq 1 \mu \mathrm{M}$, which is in agreement with our data - demonstrating a stimulation of protein synthesis in C2C12 myotubes at the same dose [16]. Our data are also in line with our previous in vivo studies demonstrating that isoflavones increased soleus muscle weight in ovariectomized female rats $[17,18]$.

Interestingly, our investigations also demonstrate that treatment with E2, SoyEx, isoflavones, and Mix resulted in a higher number of myotubes per area compared to the control cells ( $\triangleright$ Fig. $\mathbf{3 C}$ ). In all groups, the same number of cells was seeded and a comparable number of myotubes was differentiated; therefore, we speculate that in the untreated control group, myotubes may die by mechanisms related to cell death, such as apoptosis and autophagy, but this needs to be investigated in future studies in detail.

To further investigate the molecular mechanisms of soy isoflavone activity and to identify steroid hormone receptors serving as a target for SoyEx action, a co-incubation experiment with SoyEx and the antiestrogen ZK191703, or SoyEx and the antiandrogen flutamide, was performed. It is well known that C2C12 cells ex- 
A

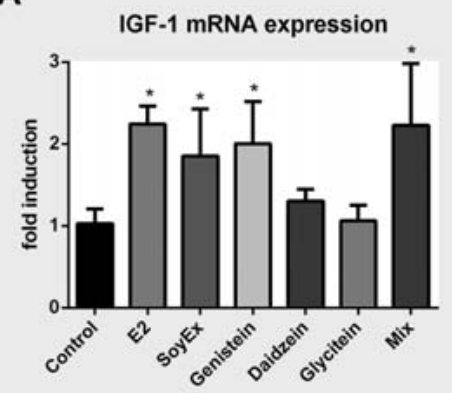

B

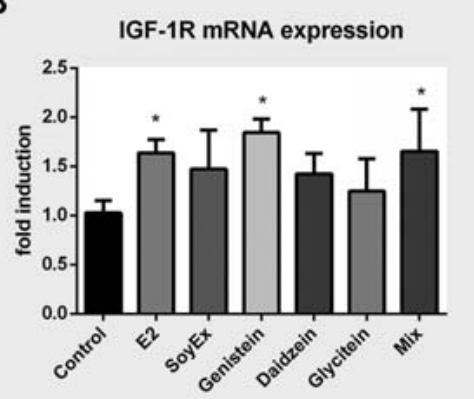

C

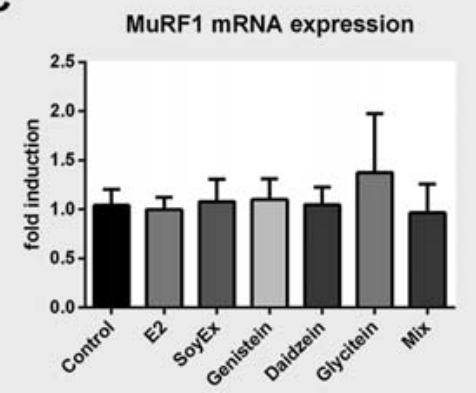

- Fig. 5 Effects of SoyEx, genistein, daidzein, glycitein, and Mix on relative mRNA expressions of IGF-1 (A), IGF-1R (B), and MuRF1 (C) in C2C12 myotubes. $\mathrm{E} 2=10 \mathrm{nM}$, SoyEx $=0.05 \mu \mathrm{g} / \mathrm{mL}$, genistein $=0.001 \mu \mathrm{M}$, daidzein $=0.0021 \mu \mathrm{M}$, glycitein $=0.0006 \mu \mathrm{M}, \mathrm{Mix}=(0.001 \mu \mathrm{M}, 0.0021 \mu \mathrm{M}$, $0.0006 \mu \mathrm{M}) .{ }^{*} \mathrm{p} \leq 0.05$ vs. control.

A

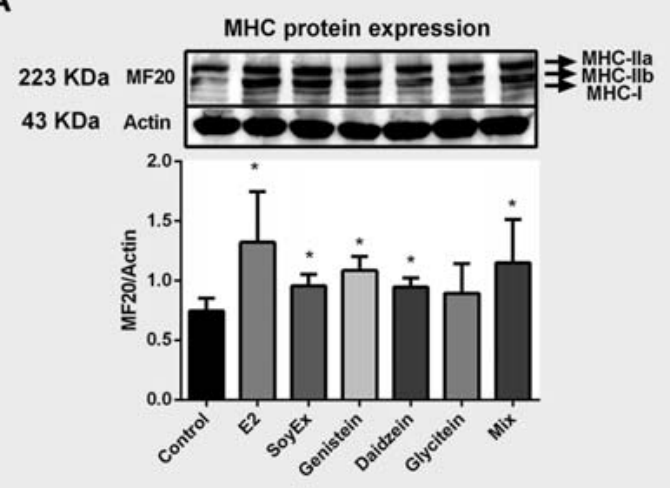

B

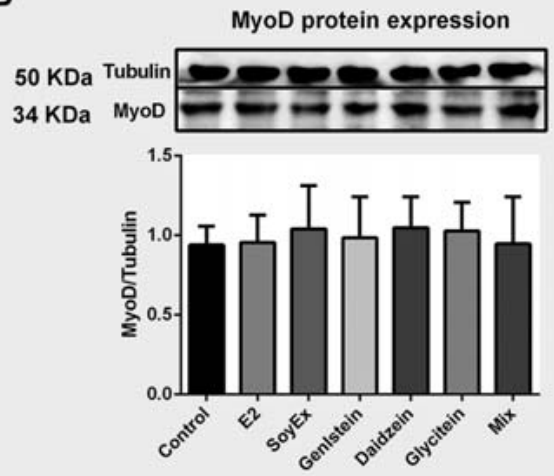

- Fig. 6 Effects of SoyEx, genistein, daidzein, glycitein, and Mix on protein expressions of MHC (A) and MyoD (B) in C2C12 myotubes. The MHC isoforms were indicated in the figure based on their molecular weights. $E 2=10 \mathrm{nM}$, SoyEx $=0.05 \mu \mathrm{g} / \mathrm{mL}$, genistein $=0.001 \mu \mathrm{M}$, daidzein $=$ $0.0021 \mu \mathrm{M}$, glycitein $=0.0006 \mu \mathrm{M}, \mathrm{Mix}=(0.001 \mu \mathrm{M}, 0.0021 \mu \mathrm{M}, 0.0006 \mu \mathrm{M}) .{ }^{*} \mathrm{p} \leq 0.05$ vs. control.

press both AR and ER $[19,20]$. In Fig. 4, it is shown that the hypertrophic effect of SoyEx can be antagonized by the antiestrogen but not by the antiandrogen, indicating the anabolic effect of SoyEx is mediated via an interaction with the ER signaling pathway rather than the AR pathway. Some studies pointed out that genistein inhibited cell growth by suppressing AR transcription in prostate cancer cell lines [21]. However, based on our results, as well as those of many other studies that have already shown that genistein binds to ER [22], we conclude that AR is not involved in promoting myotube growth induced by SoyEx.

Genistein, daidzein, and glycitein are the major isoflavone aglycones found, but isoflavone glycosides (genistin, daidzin, glycitin) were also detected in our SoyEx. In the intestine, isoflavone glycosides are first hydrolyzed and converted to corresponding bioactive aglycones [23]. It was found that the aglycones are absorbed faster and in higher amounts than their glycosides after dietary isoflavone intake in humans [24]; therefore, the anabolic effects of isoflavone aglycones and their combinations on $\mathrm{C} 2 \mathrm{C} 12$ myotubes were examined. The isoflavone aglycone mixtures contain the same amounts as analytically determined in $0.05 \mu \mathrm{g} / \mathrm{mL}$ of SoyEx ( Fig. 2B). Previously, in a study of our group, Mix did not reach the same potency as SoyEx to stimulate the proliferation of MCF-7 breast cancer cells [15]. However, in this study we found that our isoflavone aglycone mixture simulation SoyEx has a comparable anabolic potency on $\mathrm{C} 2 \mathrm{C} 12$ myotubes in vitro. Moreover, the results showed that genistein is the most potent aglycone in SoyEx with respect to anabolic activity.

The regulation of skeletal muscle mass depends on the balance between protein synthesis and degradation. The IGF-1/PI3K/Akt/ $\mathrm{PKB} / \mathrm{mTOR}$ pathway acts as a positive regulator of muscle growth [25]. Many studies have reported an important role of IGF-1 in muscle hypertrophy [26]. In addition, muscle-specific inactivation of IGF-1R impairs muscle growth due to reduced muscle fiber number and size [27]. In this study, both IGF-1 and IGF-1R mRNA expressions were detected being upregulated by SoyEx. Meanwhile, genistein increased IGF- 1 and IGF-1R expressions, and the effects can be comparable to E2, SoyEx, and Mix, indicating a strong potency of anabolic activity of genistein in soy products 
and among the isoflavone aglycones. The mRNA results of IGF-1 and IGF-1R were consistent with our data of hypertrophy. IGF-1 has been reported to stimulate muscle growth by decreasing MuRF1 [28]. MuRF1 is a muscle-specific ubiquitin ligase and regarded as an important regulator for muscle atrophy. In our study, MuRF1 gene expression was not affected by SoyEx or genistein. The explanation could be that muscle hypertrophy is independent of MuRF1 expression, which was demonstrated in another study [29].

MyoD and MHC play important roles during the process of myogenesis in C2C12 cells [30], and they are associated with myostatin expression and localization, which is known as a major negative regulator of muscle growth [31]. Our analysis of the expression of these two proteins in $\mathrm{C} 2 \mathrm{C} 12$ myotubes showed that $\mathrm{MHC}$ was affected by SoyEx, whereas MyoD was not. A possible explanation is that we analyzed myotubes in which MHC as a structural protein is part of the process of hypertrophy, whereas MyoD, which is mainly expressed during the process of differentiation, is here not of relevance anymore [30]. Several isoforms of MHC proteins are expressed in $\mathrm{C} 2 \mathrm{C} 12$ myotubes. Among them, MHC-II is strongly expressed, whereas a relatively small amount of MHC-I is expressed [31]. According to the molecular weights of $\mathrm{MHC}$ isoforms indicated in Fig. 6A, MHC-IIb might be the isoform modulated in this study. However, this needs to be further confirmed. Our previous study showed that MHC protein expressions of both soleus and gastrocnemius muscles were increased by E2 or genistein in an ovariectomized rat model [11]. In this study, the distinct effects of the compositions in isoflavone aglycones on $\mathrm{MHC}$ expression in $\mathrm{C} 2 \mathrm{C} 12$ myotubes were also investigated. The MHC expression pattern was similarly regulated as IGF-1 gene expression, which further proved the main role of genistein in SoyEx on stimulation of muscle growth. The effect was mediated by increasing MHC protein expression. Daidzein and glycitein also increased MHC protein expressions; however, the effects were weaker than those of genistein. This result was consistent with the data of IGF-1 mRNA expression and diameter measurement.

In summary, isoflavone aglycones from SoyEx induce anabolic effects on C2C12 myotubes by binding to ER and increasing IGF-1 and $\mathrm{MHC}$ expression. Among the tested aglycones, genistein seems to have the strongest anabolic bioactivity. Altogether, this study provides some evidence that SoyEx exerts beneficial effects on muscle growth.

\section{Materials and Methods}

\section{Substance and chemicals}

SoyEx was obtained from Novasoy 650 (ADM) and stored in the Department of Safety and Quality of Fruit and Vegetables, Max Rubner-Institut Karlsruhe with the lot number 0601311. Genistein, daidzein, and glycitein (purities $\geq 98 \%$ ) were purchased from LC Laboratories. E2 (purity $\geq 98 \%$ ), DHT (purity $\geq 97.5 \%$ ), and a selective antagonist of AR flutamide (purity $\geq 98 \%$ ) were purchased from Sigma-Aldrich. The antiestrogen ZK191703 (purity $\geq 98 \%$ ) was provided by Bayer Pharma AG. DMSO, EtOH, $\mathrm{MeOH}$ (purities $\geq 99.9 \%$ ), and glutaraldehyde (25\%) were purchased from Merck.
DMEM and medium components were all purchased from Gibco Life Technologies.

\section{Characterization of isoflavone derivates in SoyEx}

Qualitative and quantitative content of isoflavone derivatives in the purchased SoyEx was measured by LC-DAD analysis and performed as described previously [15,32]. In detail, SoyEx of approximately $50 \mathrm{mg}$ was weighed out accurately and vortexed for $30 \mathrm{~s}$ in exactly $40 \mathrm{~mL} 65 \%(\mathrm{v} / \mathrm{v}) \mathrm{MeOH}$. Next, samples were incubated in an overhead rotation shaker for $1 \mathrm{~h}$ at room temperature. The suspensions were centrifuged at $8600 \times \mathrm{g}$ for $5 \mathrm{~min}$ and filtered using filters with $15 \mathrm{~mm}$ diameter and $0.45 \mu \mathrm{m}$ pore size. The filtrates were diluted 50 times with $65 \%(\mathrm{v} / \mathrm{v}) \mathrm{MeOH}$ before analysis. The LC-DAD analyses were performed on a Shimadzu LC system equipped with a controller (CBM-20A), a degasser (DGU20A3), two pumps (LC-20AD), an autosampler (SIL-20AC HT), a column oven (CTO-20AC), and a diode array detector (SPDM20A). The LC system was controlled by the software LC Solution (version 1.24). Separation of the isoflavone derivatives was performed on a Phenomenex Kinetex PFP column (3.0 mm internal diameter, $100 \mathrm{~mm}$ length, $2.6 \mu \mathrm{m}$ pore size) with an oven temperature of $35^{\circ} \mathrm{C}$. Solvent $\mathrm{A}$ was $0.1 \%(\mathrm{v} / \mathrm{v})$ formic acid in bidest. water, and solvent $B$ was acetonitrile (LC grade). The flow rate was $0.7 \mathrm{~mL} / \mathrm{min}$, and the injection volume was $10 \mu \mathrm{L}$. The LC gradient started with an initial period of 3 min at $10 \%$ B, increasing linearly to $45 \%$ B at $12 \mathrm{~min}$, and finally to $100 \%$ at $12.5-15.5 \mathrm{~min}$, re-equilibrating the system in a 7.5 min postrun $(10 \% \mathrm{~B})$. Eluent was monitored between 200 and $500 \mathrm{~nm}$ using DAD. Peaks were integrated at $250 \mathrm{~nm}$. To quantify the isoflavone derivatives, external standard calibration curves for each compound in the range of $0.08-50 \mu \mathrm{M}$ were prepared. The identity of each compound was confirmed by the retention time and the UV-Vis spectra. The limit of quantification and lowest calibration point was $0.8 \mathrm{pmol}$ on column for all target analytes.

\section{C2C12 hypertrophy cell culture model}

C2C12 cells (American Type Culture Collection) were grown in phenol-red-free DMEM supplemented with $10 \%$ FBS, 4 nM glutamine, $1.5 \mathrm{~g} / \mathrm{L}$ sodium bicarbonate, $100 \mathrm{mM}$ sodium pyruvate, and 100 units/mL penicillin/streptomycin. Differentiation toward myotubes was induced at confluence by shifting the proliferation medium to differentiation medium (DMEM with $2 \%$ horse serum). During the whole experiment, the cells were stored at atmospheric conditions of $5 \% \mathrm{CO}_{2}$ and $37^{\circ} \mathrm{C}$.

Differentiated myotubes were incubated for $48 \mathrm{~h}$ in the presence of SoyEx $(0.0005-50 \mu \mathrm{g} / \mathrm{mL}), E_{2}$ (1 or $\left.10 \mathrm{nM}\right)$, ZK191703 $(1 \mu \mathrm{M})$, DHT $(1 \mu \mathrm{M})$, flutamide $(1 \mu \mathrm{M})$, genistein $(0.001 \mu \mathrm{M})$, daidzein $(0.0021 \mu \mathrm{M})$, glycitein $(0.0006 \mu \mathrm{M})$, or control $(0.1 \% \mathrm{DMSO}$ only). For the antagonization study, cells were treated with combinations of E2 $(10 \mathrm{nM})-\mathrm{ZK} 191703(1 \mu \mathrm{M})$, SoyEx $(0.05 \mu \mathrm{g} / \mathrm{ml})$ -ZK191703 $(1 \mu \mathrm{M})$, DHT-flutamide $(1 \mu \mathrm{M}$ each), and SoyEx $(0.05 \mu \mathrm{g} / \mathrm{mL})$-flutamide $(1 \mu \mathrm{M})$. Moreover, the myotubes were also treated with Mix $(0.001 \mu \mathrm{M}-0.0021 \mu \mathrm{M})$.

\section{Measurement of myotube diameters}

Cells were fixed with glutaraldehyde for $1 \mathrm{~h}$ immediately after $48 \mathrm{~h}$ incubation with the respective substances. Afterward, the myo- 
tubes were photographed using a fluorescence microscope (Axiovert $200 \mathrm{M}$, Zeiss). The glutaraldehyde induced autofluorescence after the treatment of SoyEx, E2, ZK191703, DHT, flutamide, and isoflavones. Myotube diameters were measured every $10-20 \mu \mathrm{m}$ along the length of the segments of myotubes in the photos and the average data for each myotube were calculated. Minimal 50 myotubes per group of all forms were measured by using the Axiovision LE software.

\section{Measurement of myotube number in defined areas}

Before and after treatment with the respective substances, pictures of alive cells were taken with a microscope (Axiovert $200 \mathrm{M}$, Zeiss). In order to avoid that irregular myotube growth would affect the results, pictures were taken of marked spots before chemical treatment, which could be compared to the pictures of the same spot after the treatment. At the bottom of each well, two dots were drawn with a colored marker. For each group, more than seven pictures were taken. The dots were surrounded by circles in order to find them more easily under the microscope. In the field of view of the microscope, the shadow of the circle and the dot could be detected.

\section{Real-time PCR experiments}

Total RNA was extracted from $\mathrm{C} 2 \mathrm{C} 12$ myotubes using the standard TRIzol method (Life Technologies). The quality of RNA was checked by agarose gel electrophoresis and the RNA was quantified by spectrophotometry (NanoDrop 1000, Thermo Scientific). CDNA synthesis and real-time PCR was performed as previously described [33]. The PCR program was as follows: $95^{\circ} \mathrm{C}$ for 3 min for 1 cycle, followed by 40 cycles of $95^{\circ} \mathrm{C}$ for $30 \mathrm{~s}, 59^{\circ} \mathrm{C}$ for $30 \mathrm{~s}$, $72^{\circ} \mathrm{C}$ for $30 \mathrm{~s}$, and $1 \mathrm{cycle}$ of $95^{\circ} \mathrm{C}$ for $1 \mathrm{~min}, 58^{\circ} \mathrm{C}$ for $30 \mathrm{~s}, 95^{\circ} \mathrm{C}$ for $30 \mathrm{~s}$. Fluorescence was quantified during the $59^{\circ} \mathrm{C}$ annealing step and product formation was confirmed by melting curve analysis $\left(59-95^{\circ} \mathrm{C}\right)$. Relative mRNA amounts of target genes were calculated after normalization to a housekeeping gene (GAPDH). The following oligonucleotide primers (Life Technologies) were used for amplification: GAPDH [34], fwd: 5'-ACCCAGAAGACTGTGGATGG-3', rev: 5'-TTCAGCTCTGGGATGACCTT-3'; IGF-1, fwd: 5'AGCTGCAAAGGAGAAGGAAAGGAAG-3', rev: 5'-GGTGATGTGGCATTTCCTGCT-3'; IGF-1R, fwd: 5'-GGACAACTGCCCTGATATGC3', rev: 5'-CTCCATC TCCAGCTCCTCTG-3'; MuRF1 [28], fwd: 5'AGGACAACCTCGTGCCTACAAG-3', rev: 5'-ACAACCTGTGCCGCAA GT G-3'.

\section{Western blotting}

Total protein was extracted from C2C12 myotubes using lysis buffer $(50 \mathrm{mM}$ Tris $\mathrm{pH} 8.0,2 \mathrm{mM} \mathrm{CaCl} 2,80 \mathrm{mM} \mathrm{NaCl}, 1 \%$ Triton-X $100)$ containing protease inhibitor phenylmethanesulfonylfluoride $(10 \mathrm{mM})$. Measurement of protein concentration, gel electrophoresis, and protein transfer were performed as previously described [33]. Proteins were detected using primary antibodies of mouse anti-MHC (MF 20, $223 \mathrm{KDa}$, Developmental Studies Hybridoma Bank), mouse anti-MyoD (D7F2, 34 KDa Developmental Studies Hybridoma Bank), mouse anti-actin (MAB1501, an alpha actin in skeletal muscle, $43 \mathrm{KDa}$, Millipore $\mathrm{GmbH}$ ) and mouse anti-alpha-Tubulin (12G10, 50 KDa, Developmental Studies Hybridoma Bank) overnight at $4^{\circ} \mathrm{C}$. Afterward, membranes were incubated for $1 \mathrm{~h}$ with peroxidase-conjugated secondary antibodies (rabbit anti-mouse antibody, HRP, Dako). Visualization of the blot signals was obtained by chemiluminescent reaction of the substrate luminol (Lumi-LightPlus Western Blotting Substrate, Roche) and the following detection by a fluorescent detection system (FluorChem, Alpha Innotech). ImageJ software (NIH) was used for densitometric determination of protein expression. Protein expression of MHC and MyoD was respectively related to the reference protein actin, alpha-Tubulin.

\section{Statistical analysis}

The substances were compared only to control group or between two groups as indicated in > Fig. 4. The hypotheses of statistical analysis were not for multiple testing and existed not for all pairwise comparisons. All data were expressed as means \pm standard deviation (SD). Statistical significance of differences was calculated using Kruskal-Wallis test with a subsequent Mann-Whitney U-test (GraphPad Prism version 5). Statistical significance was established at $\mathrm{p} \leq 0.05$.

\section{Acknowledgements}

This project is funded by the German Research Foundation (DFG) grant Di 716/12-1. The authors also thank Sabine E. Kulling from Department of Safety and Quality of Fruit and Vegetables, Max Rubner-Institut Karlsruhe for SoyEx composition analysis, and the China Scholarship Council for grant support to W.Z.

Conflict of Interest

The authors have declared no conflicts of interest.

\section{References}

[1] Lindle RS, Metter EJ, Lynch NA, Fleg JL, Fozard JL, Tobin J, Roy TA, Hurley BF. Age and gender comparisons of muscle strength in 654 women and men aged 20-93 yr. J Appl Physiol (1985) 1997; 83: 1581-1587

[2] Maltais M, Desroches J, Dionne I. Changes in muscle mass and strength after menopause. J Musculoskelet Neuronal Interact 2009; 9: 186-197

[3] Manson JE, Chlebowski RT, Stefanick ML, Aragaki AK, Rossouw JE, Prentice RL, Anderson G, Howard BV, Thomson CA, LaCroix AZ, Wactawski-Wende J, Jackson RD, Limacher M, Margolis KL, WassertheilSmoller S, Beresford SA, Cauley JA, Eaton CB, Gass M, Hsia J, Johnson KC, Kooperberg C, Kuller LH, Lewis CE, Liu S, Martin LW, Ockene JK, O'Sullivan MJ, Powell LH, Simon MS, Van Horn L, Vitolins MZ, Wallace RB. Menopausal hormone therapy and health outcomes during the intervention and extended poststopping phases of the Women's Health Initiative randomized trials. JAMA 2013; 310: 1353-1368

[4] Collaborators MWS. Breast cancer and hormone-replacement therapy in the Million Women Study. Lancet 2003; 362: 419-427

[5] Ososki AL, Kennelly EJ. Phytoestrogens: a review of the present state of research. Phytother Res 2003; 17: 845-869

[6] Aubertin-Leheudre M, Lord C, Khalil A, Dionne I. Six months of isoflavone supplement increases fat-free mass in obese-sarcopenic postmenopausal women: a randomized double-blind controlled trial. Eur J Clin Nutr 2007; 61: 1442-1444

[7] Yamaguchi M, Gao YH. Anabolic effect of genistein and genistin on bone metabolism in the femoral-metaphyseal tissues of elderly rats: the genistein effect is enhanced by zinc. Mol Cell Biochem 1998; 178: 377-382 
[8] Jing Y, Waxman S. Structural requirements for differentiation-induction and growth-inhibition of mouse erythroleukemia cells by isoflavones. Anticancer Res 1995; 15: 1147-1152

[9] Rommel C, Bodine SC, Clarke BA, Rossman R, Nunez L, Stitt TN, Yancopoulos GD, Glass DJ. Mediation of IGF-1-induced skeletal myotube hypertrophy by $\mathrm{PI}$ (3) K/Akt/mTOR and PI (3) K/Akt/GSK3 pathways. Nat Cell Biol 2001; 3: 1009-1013

[10] Parr MK, Zhao P, Haupt O, Ngueu ST, Hengevoss ], Fritzemeier KH, Piechotta M, Schlorer N, Muhn P, Zheng WY, Xie MY, Diel P. Estrogen receptor beta is involved in skeletal muscle hypertrophy induced by the phytoecdysteroid ecdysterone. Mol Nutr Food Res 2014; 58: 1861-1872

[11] Velders M, Solzbacher M, Schleipen B, Laudenbach U, Fritzemeier KH, Diel P. Estradiol and genistein antagonize the ovariectomy effects on skeletal muscle myosin heavy chain expression via ER-beta mediated pathways. J Steroid Biochem Mol Biol 2010; 120: 53-59

[12] Kadi F, Karlsson C, Larsson B, Eriksson J, Larval M, Billig H, Jonsdottir IH. The effects of physical activity and estrogen treatment on rat fast and slow skeletal muscles following ovariectomy. J Muscle Res Cell M 2002; 23: 335

[13] Kook SH, Choi KC, Son YO, Lee KY, Hwang IH, Lee HJ, Chung WT, Lee CB, Park JS, Lee JC. Involvement of p38 MAPK-mediated signaling in the calpeptin-mediated suppression of myogenic differentiation and fusion in C2C12 cells. Mol Cell Biochem 2008; 310: 85-92

[14] Sandri M. Signaling in muscle atrophy and hypertrophy. Physiology (Bethesda) 2008; 23: 160-170

[15] Blei T, Soukup ST, Schmalbach K, Pudenz M, Moller F], Egert B, Wortz N, Kurrat A, Muller D, Vollmer G, Gerhauser C, Lehmann L, Kulling SE, Diel P. Dose-dependent effects of isoflavone exposure during early lifetime on the rat mammary gland: studies on estrogen sensitivity, isoflavone metabolism, and DNA methylation. Mol Nutr Food Res 2015; 59: 270-283

[16] Jones K, Harty J, Roeder M, Winters T, Banz W. In vitro effects of soy phytoestrogens on rat L6 skeletal muscle cells. J Med Food 2005; 8: 327-331

[17] Kurrat A, Blei T, Kluxen FM, Mueller DR, Piechotta M, Soukup ST, Kulling SE, Diel P. Lifelong exposure to dietary isoflavones reduces risk of obesity in ovariectomized Wistar rats. Mol Nutr Food Res 2015; 59: 2407-2418

[18] Zheng W, Hengevoss J, Soukup ST, Kulling SE, Xie M, Diel P. An isoflavone enriched diet increases skeletal muscle adaptation in response to physical activity in ovariectomized rats. Mol Nutr Food Res 2017. doi:10.1002/mnfr.201600843

[19] Milanesi L, Vasconsuelo A, de Boland AR, Boland R. Expression and subcellular distribution of native estrogen receptor $\beta$ in murine $\mathrm{C} 2 \mathrm{C} 12$ cells and skeletal muscle tissue. Steroids 2009; 74: 489-497

[20] Diel P, Baadners D, Schlüpmann K, Velders M, Schwarz ]. C2C12 myoblastoma cell differentiation and proliferation is stimulated by androgens and associated with a modulation of myostatin and Pax7 expression. J Mol Endocrinol 2008; 40: 231-241

[21] Sarkar FH, Li Y. Mechanisms of cancer chemoprevention by soy isoflavone genistein. Cancer Metast Rev 2002; 21: 265-280
[22] Weigt C, Hertrampf T, Zoth N, Fritzemeier KH, Diel P. Impact of estradiol, ER subtype specific agonists and genistein on energy homeostasis in a rat model of nutrition induced obesity. Mol Cell Endocrinol 2012; 351: $227-238$

[23] Day AJ, DuPont MS, Ridley S, Rhodes M, Rhodes M], Morgan MR, Williamson G. Deglycosylation of flavonoid and isoflavonoid glycosides by human small intestine and liver $\beta$-glucosidase activity. FEBS Lett 1998; 436: 71-75

[24] Izumi T, Piskula MK, Osawa S, Obata A, Tobe K, Saito M, Kataoka S, Kubota Y, Kikuchi M. Soy isoflavone aglycones are absorbed faster and in higher amounts than their glucosides in humans. J Nutr 2000; 130: 1695-1699

[25] Schiaffino S, Dyar KA, Ciciliot S, Blaauw B, Sandri M. Mechanisms regulating skeletal muscle growth and atrophy. FEBS J 2013; 280: 4294-4314

[26] Musaro A, McCullagh K, Paul A, Houghton L, Dobrowolny G, Molinaro M, Barton ER, Sweeney HL, Rosenthal N. Localized IGF-1 transgene expression sustains hypertrophy and regeneration in senescent skeletal muscle. Nat Genet 2001; 27: 195-200

[27] Mavalli MD, DiGirolamo DJ, Fan Y, Riddle RC, Campbell KS, van Groen T, Frank SJ, Sperling MA, Esser KA, Bamman MM, Clemens TL. Distinct growth hormone receptor signaling modes regulate skeletal muscle development and insulin sensitivity in mice. J Clin Invest 2010; 120: 4007 4020

[28] Sacheck JM, Ohtsuka A, McLary SC, Goldberg AL. IGF-I stimulates muscle growth by suppressing protein breakdown and expression of atrophy-related ubiquitin ligases, atrogin-1 and MuRF1. Am J Physiol Endocrinol Metab 2004; 287: E591-E601

[29] Baehr LM, Tunzi M, Bodine SC. Muscle hypertrophy is associated with in creases in proteasome activity that is independent of MuRF1 and MAFbx expression. Front Physiol 2014; 5: 69

[30] Montesano A, Luzi L, Senesi P, Mazzocchi N, Terruzzi I. Resveratrol promotes myogenesis and hypertrophy in murine myoblasts. J Transl Med 2013; $11: 310$

[31] Artaza JN, Bhasin S, Mallidis C, Taylor W, Ma K, Gonzalez-Cadavid NF. Endogenous expression and localization of myostatin and its relation to myosin heavy chain distribution in C2C12 skeletal muscle cells. J Cell Physiol 2002; 190: 170-179

[32] Molzberger AF, Soukup ST, Kulling SE, Diel P. Proliferative and estrogenic sensitivity of the mammary gland are modulated by isoflavones during distinct periods of adolescence. Arch Toxicol 2013; 87: 1129-1140

[33] Weigt C, Hertrampf T, Kluxen FM, Flenker U, Hulsemann F, Fritzemeier $\mathrm{KH}$, Diel P. Molecular effects of ER alpha- and beta-selective agonists on regulation of energy homeostasis in obese female Wistar rats. Mol Cell Endocrinol 2013; 377: 147-158

[34] Hirasaka K, Maeda T, Ikeda C, Haruna M, Kohno S, Abe T, Ochi A, Mukai R, Oarada M, Eshima-Kondo S, Ohno A, Okumura Y, Terao J, Nikawa T. Isoflavones derived from soy beans prevent MuRF1-mediated muscle atrophy in C2C12 myotubes through SIRT1 activation. J Nutr Sci Vitaminol (Tokyo) 2013; 59: 317-324 